\title{
Integrated system modeling analysis of a cryogenic multi-cell deflecting-mode cavity resonator
}

\author{
Young-Min Shin ${ }^{1,2, a)}$ and Michael Church ${ }^{2}$ \\ ${ }^{1}$ Department of Physics, Northern Illinois University, Dekalb, Illinois 60115, USA \\ ${ }^{2}$ Fermi National Accelerator Laboratory, Batavia, Illinois 60510, USA
}

(Received 2 July 2013; accepted 12 August 2013; published online 6 September 2013)

A deflecting mode cavity is the integral element for six-dimensional phase-space beam control in bunch compressors and emittance transformers at high energy beam test facilities. RF performance of a high-Q device is, however, highly sensitive to operational conditions, in particular in a cryo-cooling environment. Using analytic calculations and RF simulations, we examined cavity parameters and deflecting characteristics of $\mathrm{TM}_{110, \pi}$ mode of a 5 cell resonator in a liquid nitrogen cryostat, which has long been used at the Fermilab A0 Photoinjector (A0PI). The sensitivity analysis indicated that the cavity could lose $30 \%-40 \%$ of deflecting force due to defective input power coupling accompanying non-uniform field distribution across the cells with $40 \sim 50 \mathrm{MeV}$ electron beam and $70-80 \mathrm{~kW}$ klystron power. Vacuum-cryomodules of the 5 cell cavity are planned to be installed at the Fermilab Advanced Superconducting Test Accelerator facility. Comprehensive modeling analysis integrated with multi-physics simulation tools showed that RF loading of $1 \mathrm{~ms}$ can cause a $\sim 5 \mathrm{~K}$ maximum temperature increase, corresponding to a $\sim 4.3 \mu \mathrm{m} / \mathrm{ms}$ deformation and a $1.32 \mathrm{MHz} / \mathrm{K}$ maximum frequency shift. The integrated system modeling analysis will improve design process of a high- $Q$ cavity with more accurate prediction of cryogenic RF performance under a high power pulse operation.

\section{INTRODUCTION}

Deflecting-mode cavities have been used in a wide range of accelerator applications that include particle separation, ${ }^{1}$ temporal beam diagnostics, ${ }^{2}$ crab-crossing in colliders, $x$-ray pulse compression, longitudinal phase space (LPS) characterization, ${ }^{3,4}$ and phase space manipulation. ${ }^{5}$ In particular, manipulation between the transverse and longitudinal phase space ${ }^{6-8}$ and single shot diagnostics of LPS $^{3}$ have been actively investigated with multi-cell deflecting mode cavities. It is a very useful tool for controlling transverse and longitudinal emittances in 6-dimensional phase space. The kick strength of the fundamental deflecting mode $\left(\mathrm{TM}_{110}\right)$ is proportional to electric and magnetic field amplitudes, which are a function of ohmic- $Q\left(Q_{0}\right)$ and transverse shunt impedance $(R / Q)$. The power dissipated in a cavity is increased with an increase in the surface resistivity, $R_{\mathrm{s}}=\left(\pi f \mu_{0} / \sigma\right)^{1 / 2}$, where $\sigma$ is the conductivity. Also, phase-matched field distribution of multi-cell structures can improve field enhancement that possibly increases shunt impedance proportional to the number of cells. Therefore, in many cases, cryogenic operation and multi-cell design are considered for deflecting mode cavities: multi-cell TESLA type cavities are made out of either normal conducting copper or superconducting niobium. As performance of the overmoded high-Q cavities are, however, highly sensitive to operational conditions, accurate control of cavity parameters is a nontrivial issue under cryocooling operation owing to the extremely narrow bandwidth of their resonance. A variety of perturbations such as mechanical vibration, microphonics, and thermal radiations

\footnotetext{
a)yshin@niu.edu
}

could significantly change characteristic cavity impedance. In particular, thermal variation resulting from cryo-cooling and RF-loading is the major source of structural deformations that bring about frequency deviations, which will therefore need to be considered in the cavity design. (The heat source of beam-loading and higher-order-modes (HOMs)/ wakefields should also be included in the analysis when cavities operate in a CW or a high rep-rate mode of high intensity beam.) However, accurate simulation modeling of a realistic operational environment is still a challenging issue for the design of a stable cryogenic RF system. Although versatile simulation technology has noticeably advanced in plasma physics and mechanical engineering, system design analysis demands complicated computational steps of data processing, which may possibly lead to the loss of data accuracy and simulation efficiency.

Over the past decade, a multi-cell deflecting $\left(\mathrm{TM}_{110}\right)$ mode cavity has been employed for phase-space manipulation tests of high brightness beams ${ }^{9-14}$ at the Fermilab A0 photoinjector (AOPI), and extended applications are currently scheduled at the Advanced Superconducting Test Accelerator (ASTA) user facility $(>50 \mathrm{MeV})$. Despite the past successful experimental results, the cavity demonstrated a limited RF performance during liquid nitrogen $\left(\mathrm{LN}_{2}\right)$ operation, which did not reach the theoretically predicted gradient. The designed cavity has been fully examined with theoretical calculations, based on the Panofsky-Wenzel theorem, using an integrated modeling tool with a comprehensive system analysis capacity to solve complex thermodynamics and the mechanical stress of the multi-cell. This paper discusses the cryogenic RF performance of the 5-cell deflecting mode cavity with numerical modeling analysis. It also presents up-to-date test simulation 


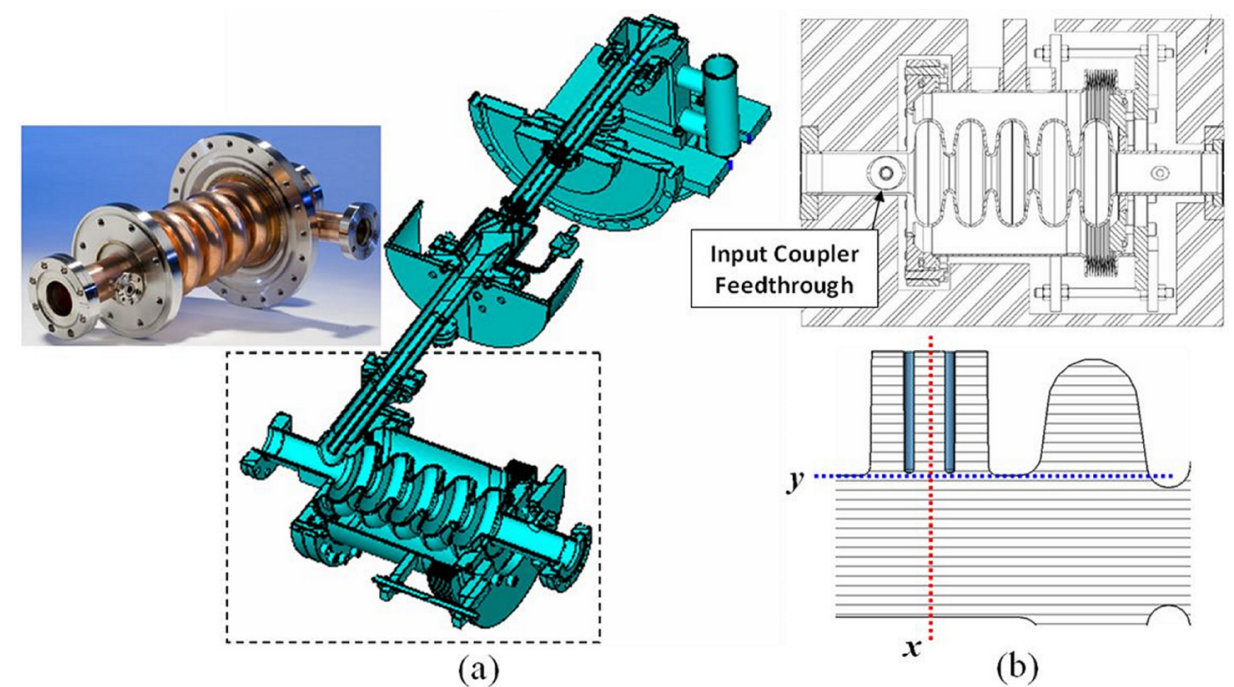

(a)

(b)
FIG. 1. (a) 3D solid model of a 5-cell deflecting mode cryomodule (inset: photo of a 5 cell, courtesy of Ref. 14) (b) top: engineering drawing; bottom: simulation model with position $(x, y)$ of coaxial antenna results of an integrated thermo-stress analysis modeling tool on the deflecting cavity vacuum-cryomodule and low power RF-test results of warm (room-temperature, 297 K) and cold ( $\mathrm{LN}_{2}$-temp, $\left.80 \mathrm{~K}\right)$ cavities.

\section{SYSTEM DESCRIPTION}

At the Fermilab A0PI, the deflecting mode cavity has been used for various beam optics experiments. As shown in Fig. 1, the cavity was designed with 5 cells to maximize kick strength and powered with a $50 \mathrm{~kW}$ (peak), S-band $(3.9 \mathrm{GHz})$ klystron. The RF power was coupled into the cavity through the high power TEM-mode coaxial coupler that was built in the $\mathrm{LN}_{2}$ vessel. The coupler design includes a temperature gradient from cryogenic temperature $(80 \mathrm{~K})$ of a $\mathrm{LN}_{2}$-ambient cavity to room temperature of an input waveguide. As the emittance exchange only requires modest fields and short pulse lengths, the $\mathrm{TM}_{110}$ mode cavity was constructed out of oxygen-free, high conductivity (OFHC) copper. ${ }^{15}$ A higher $Q_{0}$ was required than what was achievable at room temperature with the OFHC copper. Referring to $Q=\frac{2 \pi f_{0} \mu_{0} \int_{V}|H|^{2} d v}{R_{s} \int_{S}|H|^{2} d s}$ and $R_{\mathrm{S}}\left(=\left(\pi f \mu_{0} / \sigma\right)^{1 / 2}\right)$, we see that $Q_{0}$ is proportional to the square root of the copper's bulk conductivity. A $Q_{0} 2.4$ times greater was achieved by simply incorporating a $\mathrm{LN}_{2}$ cryogenic jacket into the design.

The system is designed with the $\mathrm{LN}_{2}$ vessel because the conductivity of normal conducting copper is increased 6 times from $5.8 \times 10^{7} \Omega^{-1} \mathrm{~m}^{-1}$ at room temperature to $3.5 \times 10^{8} \Omega^{-1} \mathrm{~m}^{-1}$ at $80 \mathrm{~K}$, which doubles the cavity Q. As shown in Fig. 1(b), the cryo-vessel was designed with three frequency tuning screws, attached to the chamber-outside body at one end and the flange, brazed with the beam pipe at the other end, across the flexible bellows. The tuners push the flange against the body and the mechanical pressure is transferred to the cavity through the beam pipe, so the structural distortion by the tuner induces frequency change. As this simple design did not include vacuum insulation, the $\mathrm{LN}_{2}$-temperature $(\sim 80 \mathrm{~K})$ was maintained by shielding the outer body with foam insulation. The coaxial input coupler was designed with the critical matching condition, $\beta=Q_{0} / Q_{\mathrm{e}} \sim 1$, for maximum RF power coupling into the cavity. The original design includes many practical considerations in various technical aspects. However, a high-Q cavity sensitively responds to dimensional deviations and external perturbations, which could significantly limit the deflecting performance in a cryostat. In particular, structural variation of the input coupler can significantly influence RF coupling characteristics producing an unevenly distributed field profile. It is thus highly probable that the limited deflecting performance of the 5 cell can be attributed to an off-resonance RF coupling presumably owing to design error, fabrication error, and/or cryo-cooling contraction. In order to completely identify the operational constraints, we thus investigated the cavity design and estimated its performance with a theoretical assessment incorporated with numerical data of RF simulation modeling analysis.

\section{ANALYTIC ASSESSMENT}

The integrated longitudinal acceleration of a particle traveling at the speed of light in the deflecting mode cavity is given by

$$
V_{L}(r)=\frac{r \omega}{c} \sqrt{2\left(\frac{R}{Q}\right)^{\prime} P Q_{0} \frac{4 \beta}{(1+\beta)^{2}}},
$$

where $(R / Q)$ is the shunt impedance per unit length, $P$ is the incident RF power from the klystron, $Q_{0}$ is the natural $Q$ of the cavity, $\beta$ ( $=Q_{0} / Q_{\mathrm{e}}$ ) is the coupling constant, $r$ is the

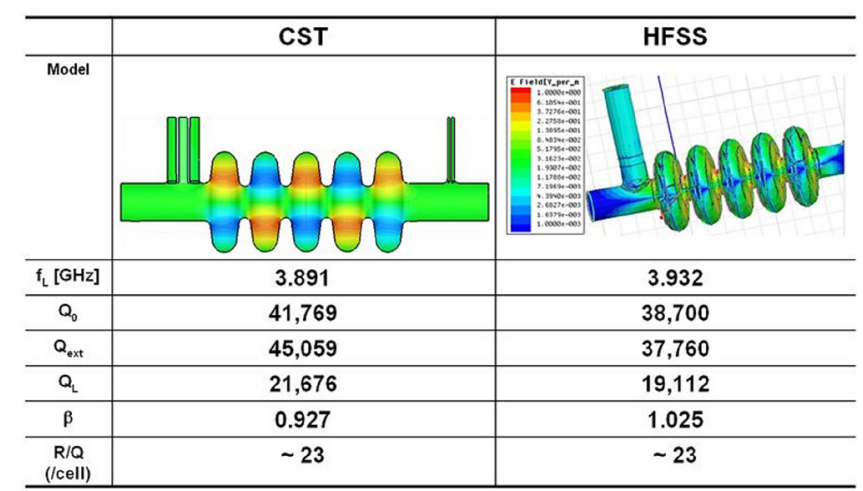

FIG. 2. (a) Field distributions and simulation data, obtained from two EM simulation $\left(3.5 \times 10^{8} \Omega^{-1} \mathrm{~m}^{-1}\right.$ at $\left.80 \mathrm{~K}\right)$. 

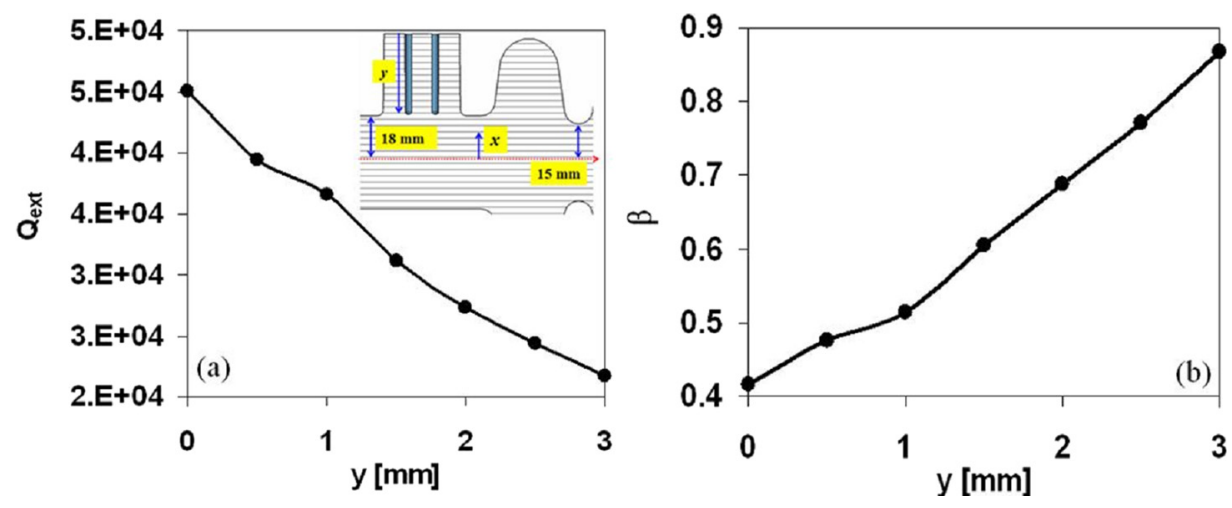

FIG. 3. (a) external $Q\left(Q_{\text {ext }}\right)$ and (b) coupling coefficient $(\beta)$ versus antenna length (y), obtained from numerical eigenmode analysis.

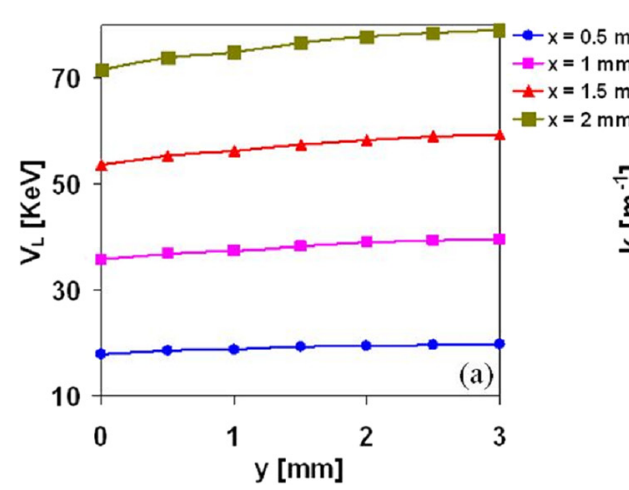

distance off axis, $\omega$ is $2 \pi$ times the resonant frequency, and $c$ the speed of light in vacuo. Here, the shunt impedance, $R / Q$, is calculated from field data obtained by RF simulation. ${ }^{16}$ Figure 2 summarizes the simulation results of the $\mathrm{TM}_{110}$ mode cavity from two RF computational codes, CST and HFSS. The two sets of the cavity parameters agree reasonably well. In particular, coupling constants are each close to critical coupling $(\beta \sim 1)$. (For the calculation, the copper conductivity was defined as $\sigma=3.5 \times 10^{8} \Omega^{-1} \mathrm{~m}^{-1}$ at $\mathrm{LN}_{2}$ temperature, $\sim 80 \mathrm{~K}$ ).

This optimized cavity design was numerically investigated by adjusting matching impedance of the coupler: scanning the radial distance from the cavity axis, $x$, and the relative length of the coaxial antenna, $y$ (Fig. 3(a)). The beam pipe radius $(18 \mathrm{~mm})$ is $3 \mathrm{~mm}$ larger than the cavity iris radius $(15 \mathrm{~mm})$. One can see that external coupling through the coupler becomes stronger as its antenna becomes longer and its tip gets closer to the axis. Here, $y=0 \mathrm{~mm}$ indicates the "original design position" of the coaxial antenna tip. As shown in Fig. 3 (b), the original coupler design $(y=0 \mathrm{~mm})$ only has $\beta=0.42$, but it is increased to $\beta=0.87$ with $\mathrm{y}=3 \mathrm{~mm}$.

Figure 4 shows the longitudinal acceleration potential $\left(V_{\mathrm{L}}\right)$ versus antenna length $(y)$ plots with respect to the radial position $(x)$ of a particle. For this calculation, we used the same beam and RF parameters listed in Fig. 2 with $16 \mathrm{MeV}$ beam energy and $50 \mathrm{~kW}$ klystron power. In Fig. 4(a), the increment of the potentials $\left(V_{\mathrm{L}}\right)$ of $y=0 \mathrm{~mm}$ and $\mathrm{y}=3 \mathrm{~mm}$ is raised from $2 \%$ of $x=0.5 \mathrm{~mm}$ to $15 \%$ of $x=2 \mathrm{~mm}$., which enhances the deflecting force, as shown in Fig. 4(b), i.e., kick strength is increased from $\sim 2.4$ at $\mathrm{y}=0 \mathrm{~mm}$ to $\sim 2.64$ at $\mathrm{y}=3 \mathrm{~mm}$, which corresponds to a $10 \%$ increment. We extensively examined the deflecting force with field uniformity and RF-coupling coefficient in the analysis (Fig. 5). The two main parameters were examined with respect to klystron

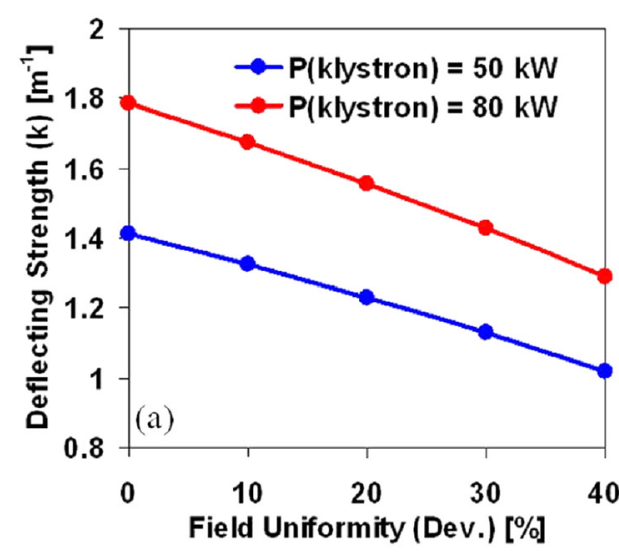

Field Uniformity (Dev.) [\%]

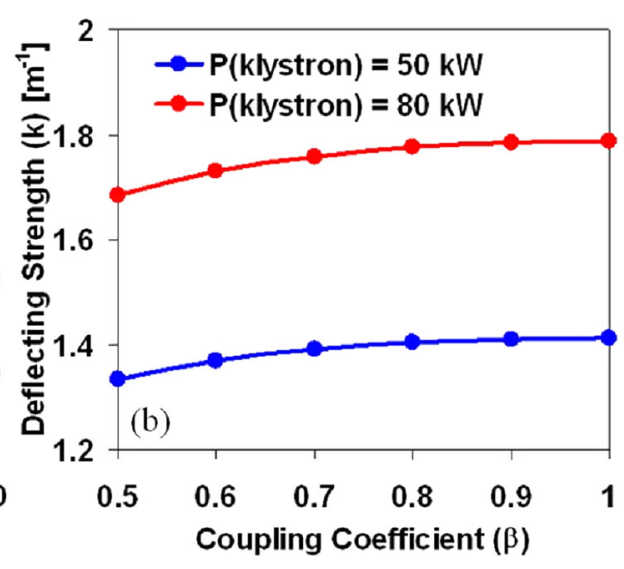

FIG. 5. Deflecting force versus (a) field non-uniformity (deviation) and (b) coupling coefficient $(\beta)$, obtained from analytic calculation with eigenmode simulations. Beam energy is $40 \mathrm{MeV}$. 

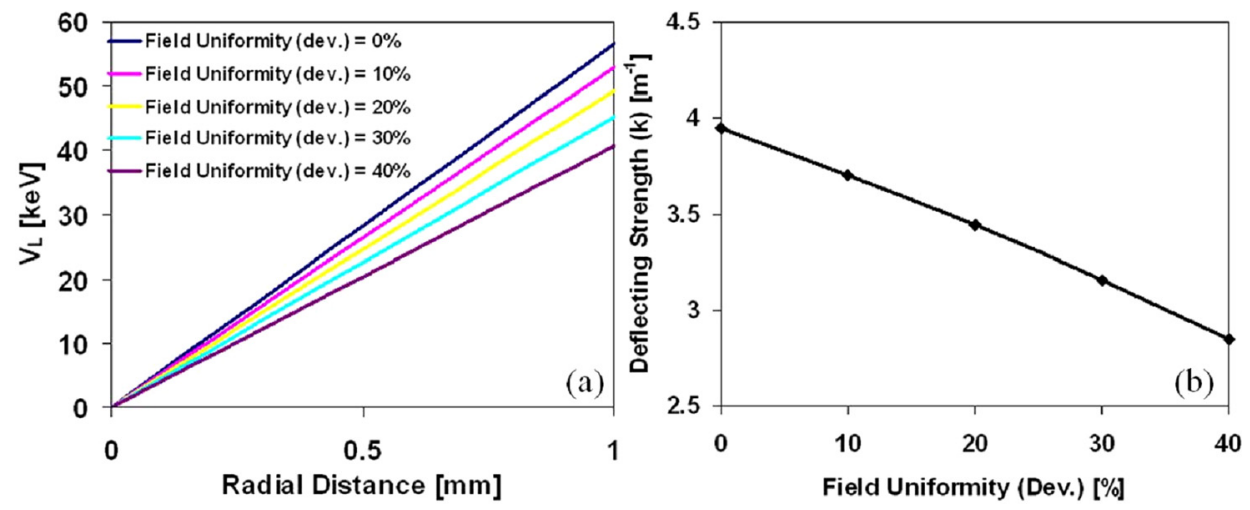

FIG. 6. (a) Deflecting voltage (integrated longitudinal acceleration field) versus radial distance graph with respect to field non-uniformity (b) deflecting force versus field nonuniformity graph, obtained from analytic calculations with eigenmode simulations. Beam energy is $40 \mathrm{MeV}$. input driving powers of $50 \mathrm{~kW}$ and $80 \mathrm{~kW}$, as a new, pulsed $80 \mathrm{~kW}, 3.9 \mathrm{GHz}$ Varian model VA-908K2 klystron has become available. Figure 5 shows that a $40 \%$ loss of field uniformity drops the deflecting force, $k$, down to 1.1 from 1.4 and to 1.4 from 1.8 with $50 \mathrm{~kW}$ and $80 \mathrm{~kW}$ of klystron power, respectively. This corresponds to a $\sim 27 \%$ reduction of the deflecting force with $50 \mathrm{~kW}$ klystron power, whereas RF driving with $80 \mathrm{~kW}$ can improve it by $27 \%$. The influence of the coupling constant, $\beta$, on the deflecting force is relatively small; i.e., $k=1.4(50 \mathrm{~kW})$ and $1.8(80 \mathrm{~kW})$ at $\beta=1 \rightarrow \mathrm{k}=1.35(50 \mathrm{~kW})$ and $1.7(80 \mathrm{~kW})$ at $\beta=0.5$ : only a $3.7 \%$ and $5.8 \%$ drop. We conclude that the offresonance coupling leads to a reduction in the kick strength because it more likely perturbs the field distribution, decreasing cell-to-cell field uniformity, rather than directly weakening the field strength. Figure 6 shows the accelerating potential vs. radial position and the deflecting force versus the field deviation. Note that the increasing rate of the deflecting field amplitude along the radial distance is noticeably reduced with loss of field uniformity, which is reflected in the deflecting force: kick strength drops down to 2.9 from 3.95 with $40 \%$ non-uniformity $(35.6 \%$ reduction). Eventually, one can see that poorly coupled RF power, accompanying perturbation of the field distribution, possibly causes a $30 \%-40 \%$ deficiency of the deflecting strength.
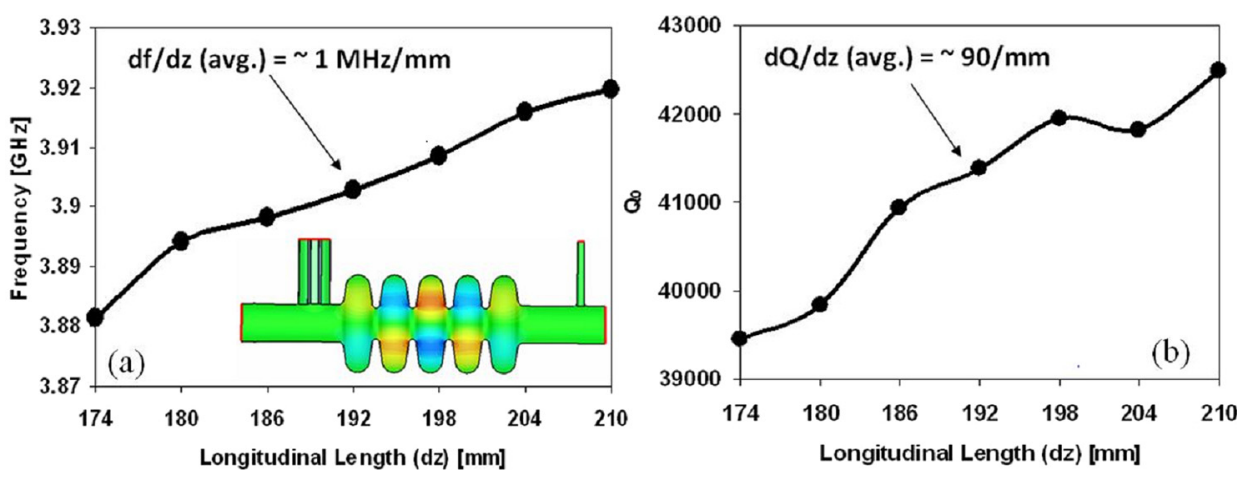

FIG. 7. (a) Loaded cavity resonant frequency and (b) ohmic $Q\left(Q_{0}\right)$ of operating mode $\left(\pi, \mathrm{TM}_{110}\right)$ versus longitudinal length ( 5 cell cavity) graph.

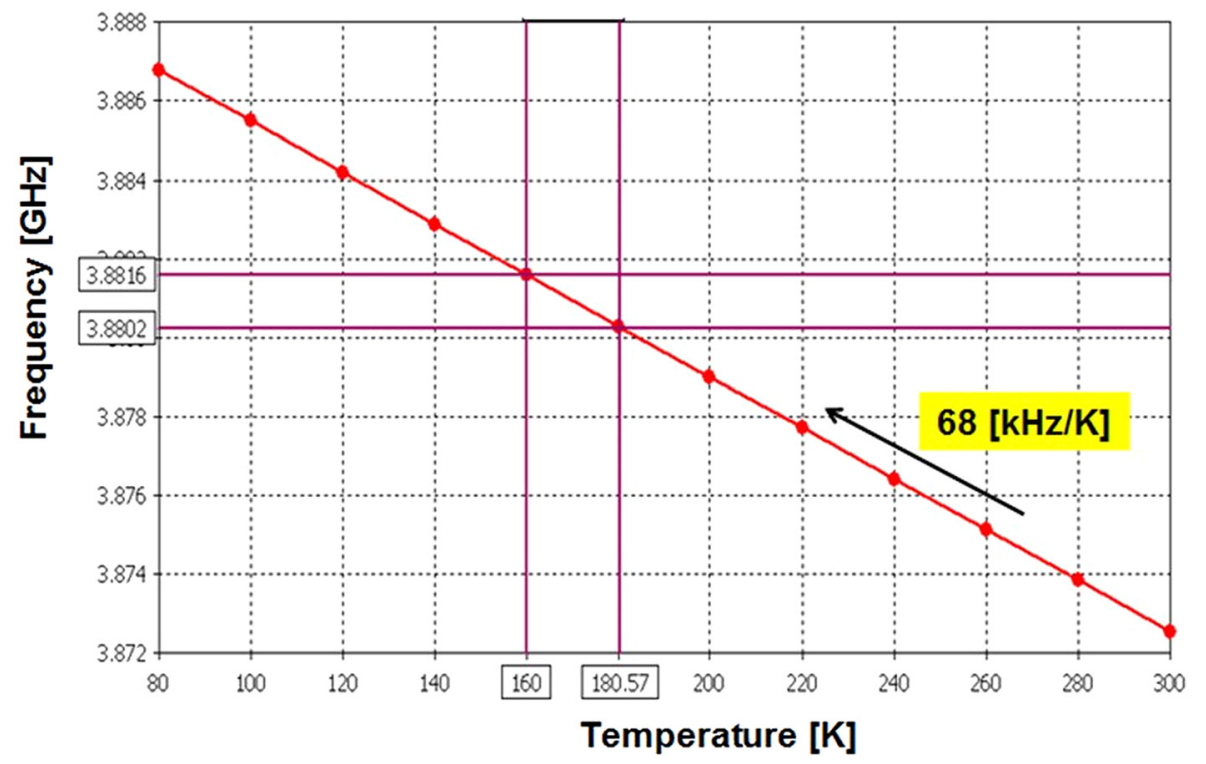

FIG. 8. Frequency versus temperature graph, obtained from eigenmode simulations using linear thermal expansion coefficient $(\alpha)$. 


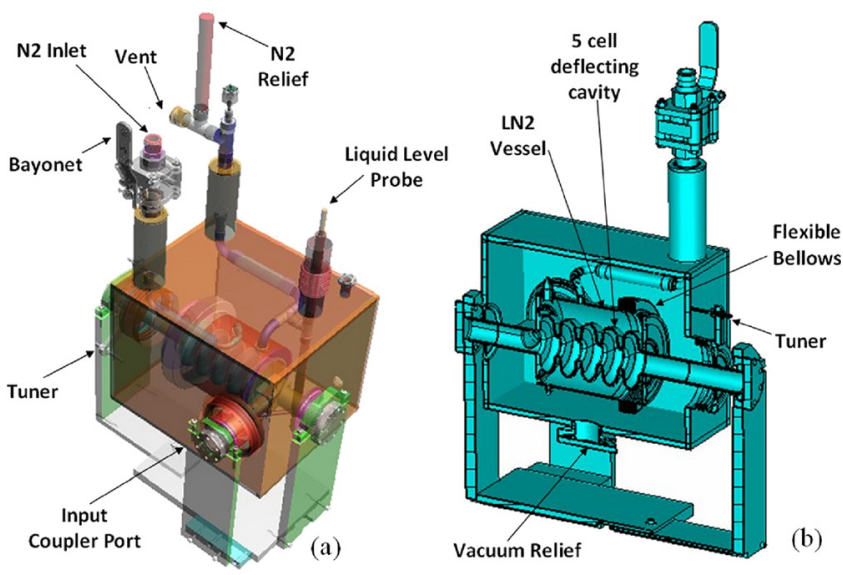

FIG. 9. Designed vacuum-cryostat with a 5 cell deflecting mode cavity (a) $\mathrm{X}$-ray image and (b) cross-sectional view.

The influence of temperature variation on the cavity parameters has also been examined by scanning the dimensional scale of the cavity geometry. Under a heavy thermal expansion, a multi-cell cavity would experience severe stress with major deformation in the longitudinal direction as the cavity is designed to be mechanically constrained at the two ends. Thus, we first considered a simple model, which imposes a dimensional change only to the cavity length. Figure 7 shows the frequency and $Q_{0}$ versus longitudinal cavity length graphs. The average deviation of the loaded $\pi$-mode frequency and $Q_{0}$ is respectively $\sim 1 \mathrm{MHz} / \mathrm{mm}$ and $\sim 90 / \mathrm{mm}$, which is small compared to typical frequency shifts in experiments. This thermal analysis model is somewhat impractical as a cavity resonant frequency depends mostly on the radial dimension. Besides, it is not easy to directly convert a 3-dimensional thermal effect to a one dimensional linear expansion. Therefore, we translated the impact of temperature change into the cavity with a linear thermal expansion coefficient of copper $(1.66 \mu \mathrm{m} / \mathrm{m} / \mathrm{K})$ by adjusting the volumetric scaling factor of the structural dimensions.
Figure 8 shows the frequency shift $\left(\mathrm{TM}_{110, \pi}\right.$ mode $)$ due to the effective temperature declination from room temperature $(300 \mathrm{~K})$ to $\mathrm{LN}_{2}(80 \mathrm{~K})$, which is explained by the uniform change of the cavity volume. Note that the frequency is increased from $3.8725 \mathrm{GHz}$ at $300 \mathrm{~K}$ to $3.8870 \mathrm{GHz}$ at $80 \mathrm{~K}$, which corresponds to $68 \mathrm{kHz} / \mathrm{K}$. The simulation analysis estimates that cryo-cooling causes roughly a $24 \mathrm{MHz}$ frequency deviation to the design cavity that would need to be considered in the process of determining cavity dimensions. This approximate analytic assessment is effective for quantifying an average deviation with the assumption that the entire cavity uniformly contracts with a decrease in temperature. However, it does not accurately predict local displacements due to RF-loading impacts over the structure, in particular on the system with fixed points. Moreover, it is limited in accurately implementing localized effects of critical heat sources such as RF loading and beam loading in the cryomodule analysis. Therefore, we extensively investigated RF-thermal characteristics of this 5-cell cryomodule with full 3D thermal and mechanical simulation modeling analysis. More details are discussed in Sec. IV.

\section{SIMULATION MODELING}

Figure 9 depicts the recently designed vacuumcryomodule containing the $3.9 \mathrm{GHz}$ 5-cell deflecting mode cavity that could be installed at the Fermilab ASTA user's facility. The system design looks very similar to the one depicted in Fig. 1, although it is designed with the vacuum insulator, instead of the foam insulator, which has much better thermal insulation efficiency. Volumetric contraction and thermal fluctuation resulting from $\mathrm{LN}_{2}$ cooling is no longer a critical factor since the vacuum insulator tank is an excellent heat reservoir for the $\mathrm{LN}_{2}$ vessel, which linearly cools the ambient temperature down to $77-80 \mathrm{~K}$ and maintains it consistently. Instead, it is more critical to consider RF loading and beam loading for $\mathrm{CW}$ or high duty operation of high intensity or high power machines. The frequency tuner is
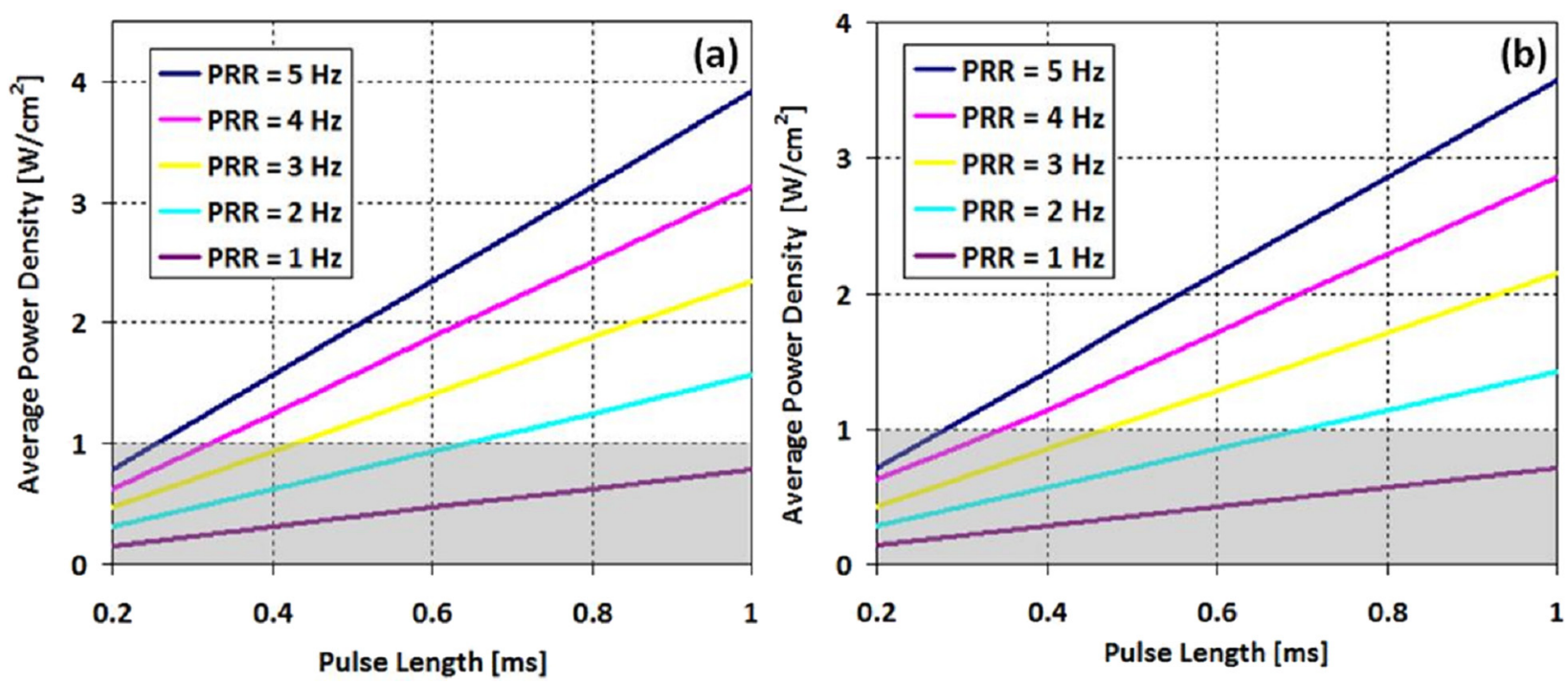

FIG. 10. Average power density deposited on the (a) internal and (b) external cavity surfaces versus RF pulse length with respect to PRR. 


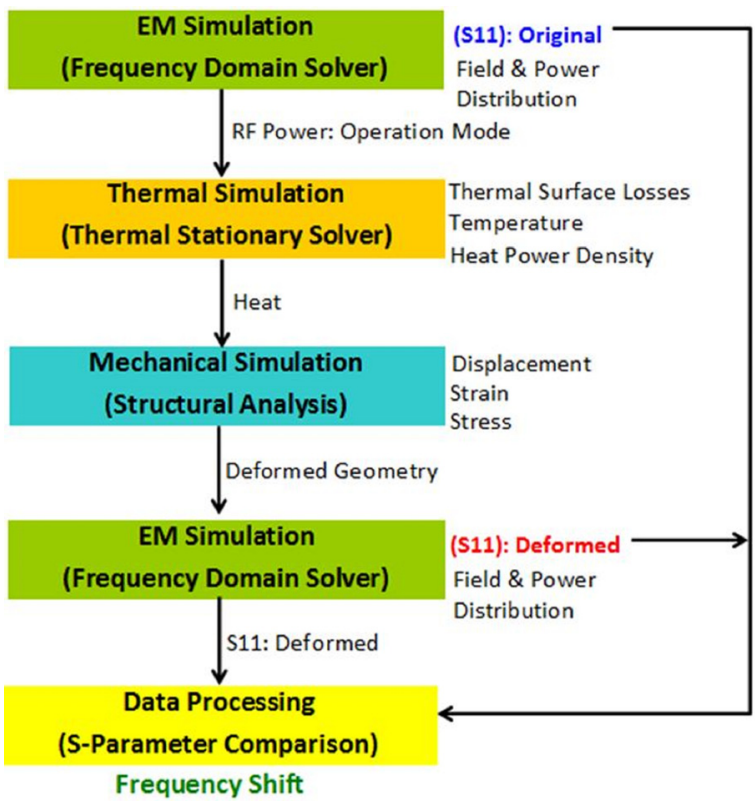

FIG. 11. Flow chart of integrated thermal and mechanical simulation batch process analysis.

similar to the original design except that it is mounted on the vacuum tank with an additional flexible bellows. In this design, the amount of liquid nitrogen is controlled by the $\mathrm{N}_{2}$ inlet and vent/relief and its level is monitored by the $\mathrm{LN}_{2}$ level probe. The temperature of the $\mathrm{LN}_{2}$ vessel remains constant by means of the vacuum insulation tank. However, even in the thermally insulated vacuum system, energy of long pulse duration deposits a considerable amount of power on the cavity surfaces that can increase the ambient temperature so highly as to exceed the temperature threshold of bubble formation in the $\mathrm{N}_{2}$ fluid.

If a deposited power density on the cavity surface exceeds $1 \mathrm{~W} / \mathrm{cm}^{2}$ on average, it is highly probable that the ambient temperature exceeds the $\mathrm{LN}_{2}$ boiling point $(\sim 77-80 \mathrm{~K})$ so as to rapidly increase gap pressure and create $\mathrm{N}_{2}$ bubbles that may change the ambient pressure around the cavity in the $\mathrm{LN}_{2}$ vessel. The excessive fluctuation of vessel pressure could substantially impact cavity performance via frequency change, structural distortion, and even quench on the cavity. Therefore, we simply calculated average power densities deposited on the cavity surface with respect to pulse width and pulse repetition rate (PRR). For the calculation, it is assumed that the cavity receives $70 \mathrm{~kW}$ from the klystron and the surface area of the 5 cell is $97.96 \mathrm{~cm}^{2}$ outside and $89.36 \mathrm{~cm}^{2}$ inside, respectively. In Fig. 10, the gray areas denote a pulse condition with less than $1 \mathrm{~W} / \mathrm{cm}^{2} \mathrm{RF}$ deposition on the outside (a) and inside (b) cavity surface. Although the calculation is based on the assumption that total RF power is deposited on the cavity surface, it appears that the RF pulse condition $(1 \mathrm{~ms} / 5 \mathrm{~Hz})$ of $3.9 \mathrm{GHz}$ deflecting mode cavity planned for the ASTA test facility surely exceeds $1 \mathrm{~W} / \mathrm{cm}^{2}$, which is about $4 \mathrm{~W} / \mathrm{cm}^{2}$ inside and $3.5 \mathrm{~W} / \mathrm{cm}^{2}$ outside. We examined the RF-loading characteristics with a full 3D simulation modeling analysis using an integrated multiphysics computational platform.

Figure 11 shows the flowchart of a batch-processing simulation algorithm for sensitivity analysis. The comprehensive sensitivity analysis modeling process starts with an electromagnetic (EM) simulation using a frequency domain solver to solve S-parameter through the input coupler. The RF surface loss of a single frequency signal obtained from a field monitor is transferred to the stationary thermal solver that calculates thermal surface losses, temperature distribution, and heat power density. From the heat, source of the thermal solver, the mechanical stress solver calculates structural displacement (deformation), strain, and stress, including a Van Mises stress analysis. The deformed geometry configuration is transferred to a frequency domain solver again, which calculates the S-parameter spectrum of the RF-loaded cavity structure. As a final step, the calculated S-parameter spectrum is compared with the original one to accurately quantify a frequency shift per RF-loading.

As shown in Figure 12(b), a broad frequency sweep of the frequency domain solver shows the $\mathrm{TM}_{110, \pi}$ mode appearing at $\mathrm{f}=3.876 \mathrm{GHz}$ with an insertion loss of $-25 \mathrm{~dB}$. There are the other resonances, though not present in Fig. 12(b), in the $S_{11}$ spectrum, corresponding to higher order $\mathrm{TM}_{110}$ modes $(3 / 4 \pi, 1 / 2 \pi$, and $1 / 4 \pi)$. The total $Q\left(Q_{\mathrm{L}}\right)$ of the operational mode is calculated to be $\sim 20000$ and relative RF-phase is $\sim 29.75^{\circ}$ at the $\mathrm{TM}_{110}$-mode resonance. The inset of Fig. 12(a) shows the field distribution of the power fed into the cavity through the input coupler. The RF data are transferred to the thermal stationary solver, as depicted in Fig 11, and the thermal losses are calculated with OFHC copper conductivity $\left(\sigma=3.5 \times 10^{8} \mathrm{~S} / \mathrm{m}\right)$ at $80 \mathrm{~K}$, and the calculated data are exported to the thermal stationary solver. Figure 13(a) shows 2D and 3D spatial distributions of temperature increase with a $1 \mathrm{~ms}$ and $5 \mathrm{~Hz}$ pulse of $80 \mathrm{~kW}$ klystron power.
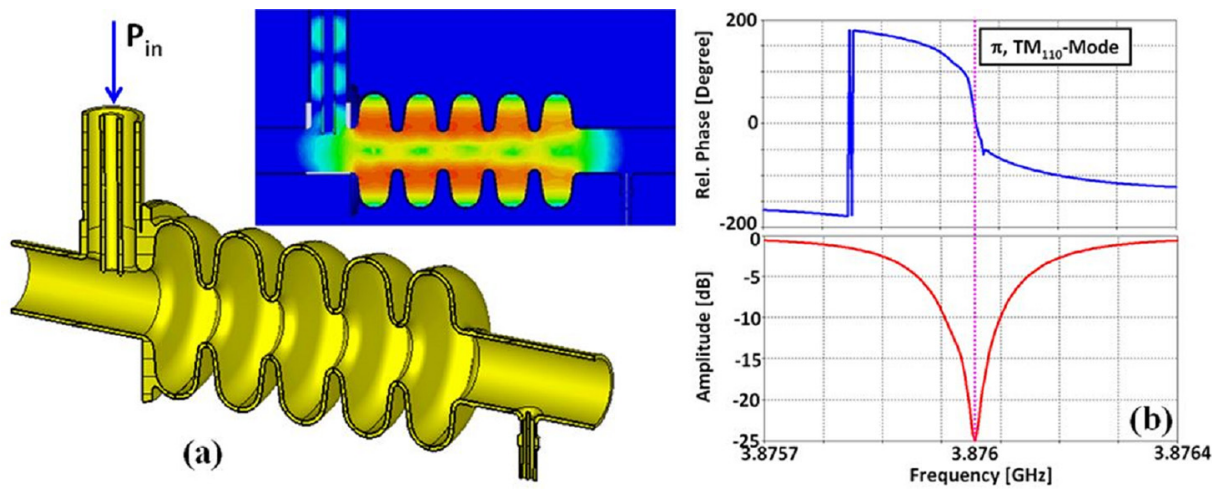

FIG. 12. Frequency domain simulation result (a) power distribution of $\mathrm{TM}_{110, \pi}$ mode (b) $\mathrm{S}_{11}$ spectrum. 

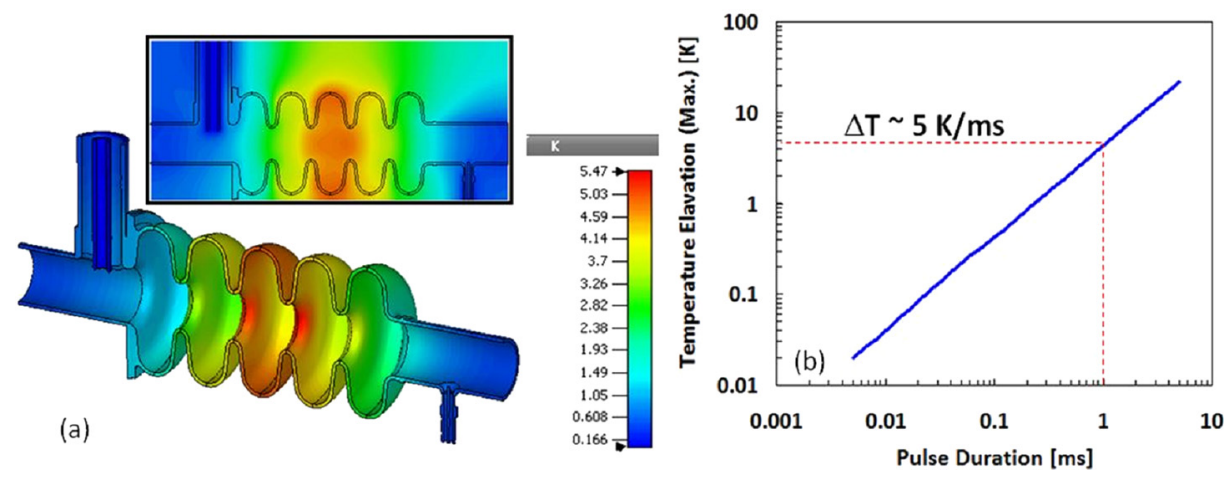

FIG. 13. Thermal stationary simulation result with transferred $\mathrm{RF}$ data from Fig. 12(a) 3D temperature distribution plot and (b) temperature elevation versus pulse duration.

For the simulation, thermal surface property is defined with an emissivity of 0.7 and a heat transfer coefficient of $300 \mathrm{~W} / \mathrm{m}^{3} / \mathrm{K}$ on the inner and outer cavity surfaces and all the thermal boundaries are supposed to have an adiabatic interface $(d Q=0)$ with consistent heat transfer. Note that power deposition is concentrated on the middle cell with the largest temperature increase, which is projected from a field distribution of the $\mathrm{TM}_{110, \pi}$ mode. Figure 13(b) shows the maximum temperature elevation versus pulse duration graph, obtained from a thermal simulation with $5 \mathrm{~Hz}$ PRR and $80 \mathrm{~kW}$ klystron power. This operation condition will eventually raise the ambient temperature up to $\sim 85 \mathrm{~K}$ which exceeds the $\mathrm{LN}_{2}$ boiling point. In order to keep the temperature below the nitrogen boiling point, the $\mathrm{LN}_{2}$ flow rate in the vessel should remain high enough to maximize cooling efficiency. The parametric analysis of the RF pulsing condition was done with sweeping pulse widths and PRRs.

Figure 14 depicts a 3D contour plot of stress (maximum Von Mises) distribution over the cavity surface and geometrical displacement of deformed structure, calculated from transferred thermal distribution data. Under the same pulse condition $(1 \mathrm{~ms} / 5 \mathrm{~Hz})$, the simulation results in maximum Von Mises stress of $0.005971 \mathrm{GPa}$, leading to an $\sim 4.36 \mu \mathrm{m}$ maximum displacement (bottom in Fig. 14(a): 10 times magnified figure). Once can see that the irises undergo the highest pressure, while maximum deformations appear at the equators. In this simulation, as depicted in the figure, the structure analysis model is programmed with three fixed points at the entrance and exit of the beam pipe and input coupler/probe coupler.

Figure 14(b) shows the dimensional deformation versus pulse duration graph, obtained from this structural modeling analysis. Note that pulse widths below $10 \mu$ s do not cause a detectable change of dimensional deformation (max.) on cavity geometry. Deformation steeply increases with pulse width above $100 \mu \mathrm{s}$, which causes $0.2 \sim 4.3 \mu \mathrm{m}$ displacement (max.) with up to $1 \mathrm{~ms}$. A frequency shift due to the structural deformation will cause a substantial change in the coupler matching condition, which can create an off-resonance power coupling with a huge reflection. Figure 15 shows $S_{11}$ spectra of the original and deformed 5 cell structures, obtained from frequency domain solvers before and after RF-loading of $1 \mathrm{~ms}$ pulse to the cavity. The displacement of thermal energy deposition causes $7.32 \mathrm{MHz}$ frequency shift, accompanied with $2.82 \mathrm{~dB}$ amplitude change and $59^{\circ}$ phase deviation, on the $\mathrm{TM}_{110, \pi}$ mode, which is beyond the FWHM (full-width-halfmaximum) bandwidth of the input signal. The summarized simulation results show that the designed system has a thermal sensitivity of $1.32 \mathrm{MHz} / \mathrm{K}$, corresponding to $0.51 \mathrm{~dB} / \mathrm{K}$ and $10.7^{\circ} / \mathrm{K}$. This correction resulting from RF-loading should thus be included in cavity design for enabling optimal operation and accurate control of the vacuum-cryomodule. In addition, the beam-loading effect could also be another heat source capable of creating a critical thermal impact with $\mathrm{CW}$ or long pulse operation. However, the beam power might have a trivial influence with $<1 \mathrm{~ms}$ and $<5 \mathrm{~Hz}$ condition.

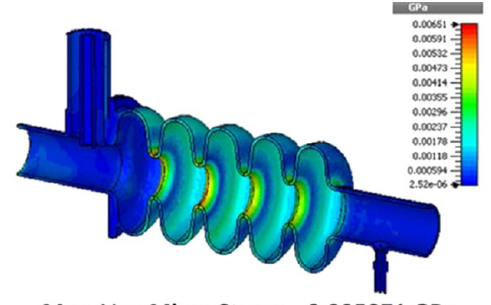

Max. Van Mises Stress $=0.005971 \mathrm{GPa}$ Fixed Point

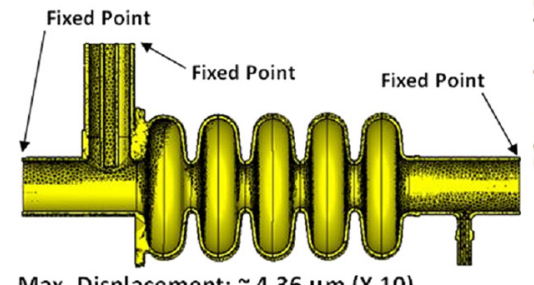

Max. Displacement: 4.36 $\mu \mathrm{m}$ (X 10)

(a)

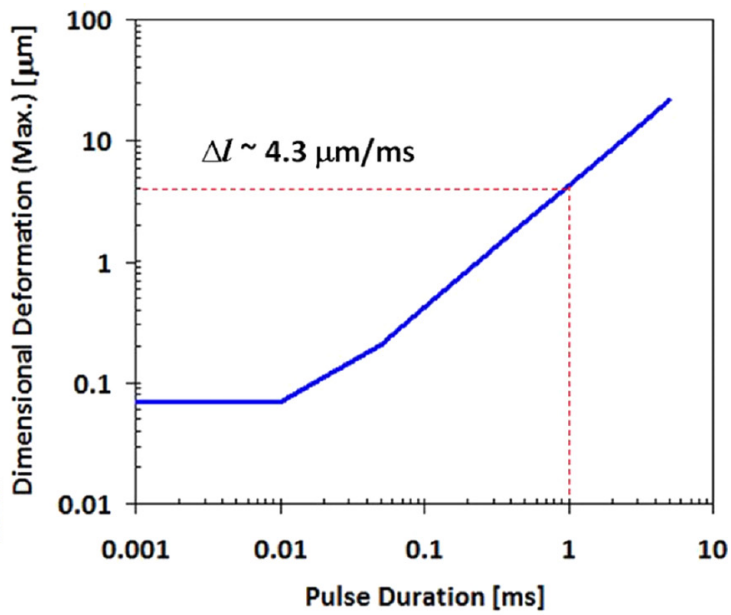

(b)
FIG. 14. Mechanical structural simulation results with transferred thermal loss data of Fig. 13. (a) 3D plot of Von Mises stress (b) geometrical displacement (structural deformation), and (c) maximum dimensional deformation versus pulse duration graph with $5 \mathrm{~Hz}$ PRR. 


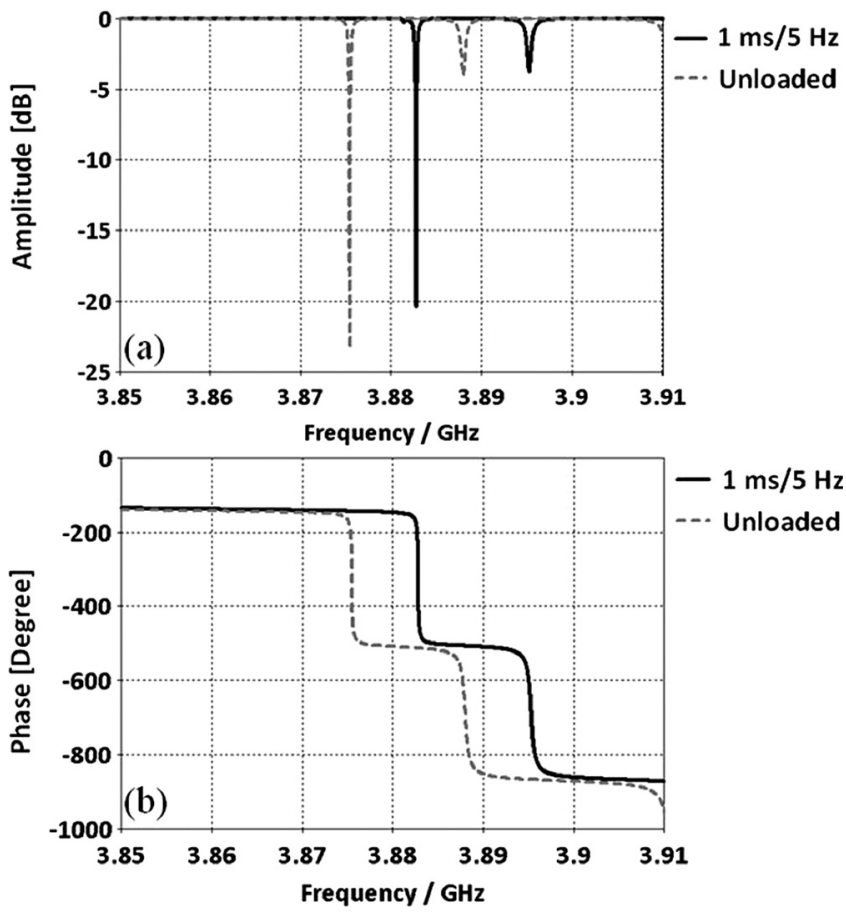

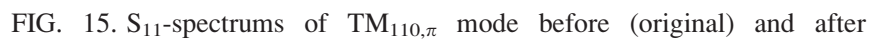
(deformed) RF-loading of $1 \mathrm{~ms}$ pulse duration and $5 \mathrm{~Hz}$ PRR, calculated from the deformed structure of Fig. 14.

\section{EXPERIMENTAL TEST}

The 5 cell cavity in the cryo-vessel, after completely shielded by thermal insulation foams, was tested at room$(297 \mathrm{~K})$ and $\mathrm{LN}_{2}$-temperature $(80 \mathrm{~K})$. The cold cavity parameters were measured twice after 0 day and 20 days long cooling operations to observe temporal variation of thermal stability. Figure 16 shows $S_{11}$ spectra (amplitude and phase) of the warm and cold cavities and thermal variations of a $\mathrm{TM}_{110}$-mode. It appears that the resonant frequency is upshifted with $\sim 12.1 \mathrm{MHz}$ by the thermal transition from
$\mathrm{T}=297.2 \mathrm{~K}$ to $\mathrm{T}=80 \mathrm{~K}$, corresponding to $55.74 \mathrm{kHz} / \mathrm{K}$, under the condition that cavity frequency varies linearly with temperature. The resonant frequency further rose to $3.899922 \mathrm{GHz}$ after 20 days cooling, corresponding to converged thermal frequency variation of $\sim 57 \mathrm{kHz} / \mathrm{K}$, which is close to the simulation result, $64 \mathrm{kHz} / \mathrm{K}$ (Fig. 8). The return loss remains steady at the initial cooling, while it is gradually increased to $\sim-10 \mathrm{~dB}$, corresponding to $0.0137 \mathrm{~dB} / \mathrm{K}$. Even the reduction of coupling level will leave the system fairly operational as $90 \%$ of driving power is still coupled in the system. The instantaneous phase deviation is $\sim 0.18^{\circ} / \mathrm{K}$, but it is also gradually increased up to $0.447^{\circ} / \mathrm{K}$ after 20 days cryo-operation. Therefore, the measurement implies that in the early stage of $\mathrm{LN}_{2}$-operating mode, the system can operate with temporal change of $<5 \mathrm{~K}$ in terms of amplitude/ phase deviation. However, as the vessel loses cooling efficiency with increasing time, acceptable temperature range for the system drops down to $<2 \mathrm{~K}$, which might exceed ambient thermal fluctuation beyond controllable range of the foam-insulated system. For a better thermal management, a cryo-vessel should thus be designed with a vacuuminsulation, which is currently planned with the ASTA 5-cell deflecting mode cavities.

\section{CONCLUSION}

A normal conducting multi-cell deflecting mode cavity has been used for various beam control applications in an $\mathrm{LN}_{2}$-vessel at the Fermilab A0PI and currently it is planned to accommodate vacuum-cryomodules of either normal conducting or superconducting deflecting mode cavities in the ASTA beam line for higher energy beam tests. Despite successful test results of the previous experiments on $6 \mathrm{D}$ phasespace manipulation, the 5-cell cavity demonstrated limited performance. Theoretical and numerical investigations of the $\mathrm{LN}_{2}$-ambient operational parameters showed that a reduction of kick strength could be ascribed to an improper impedance
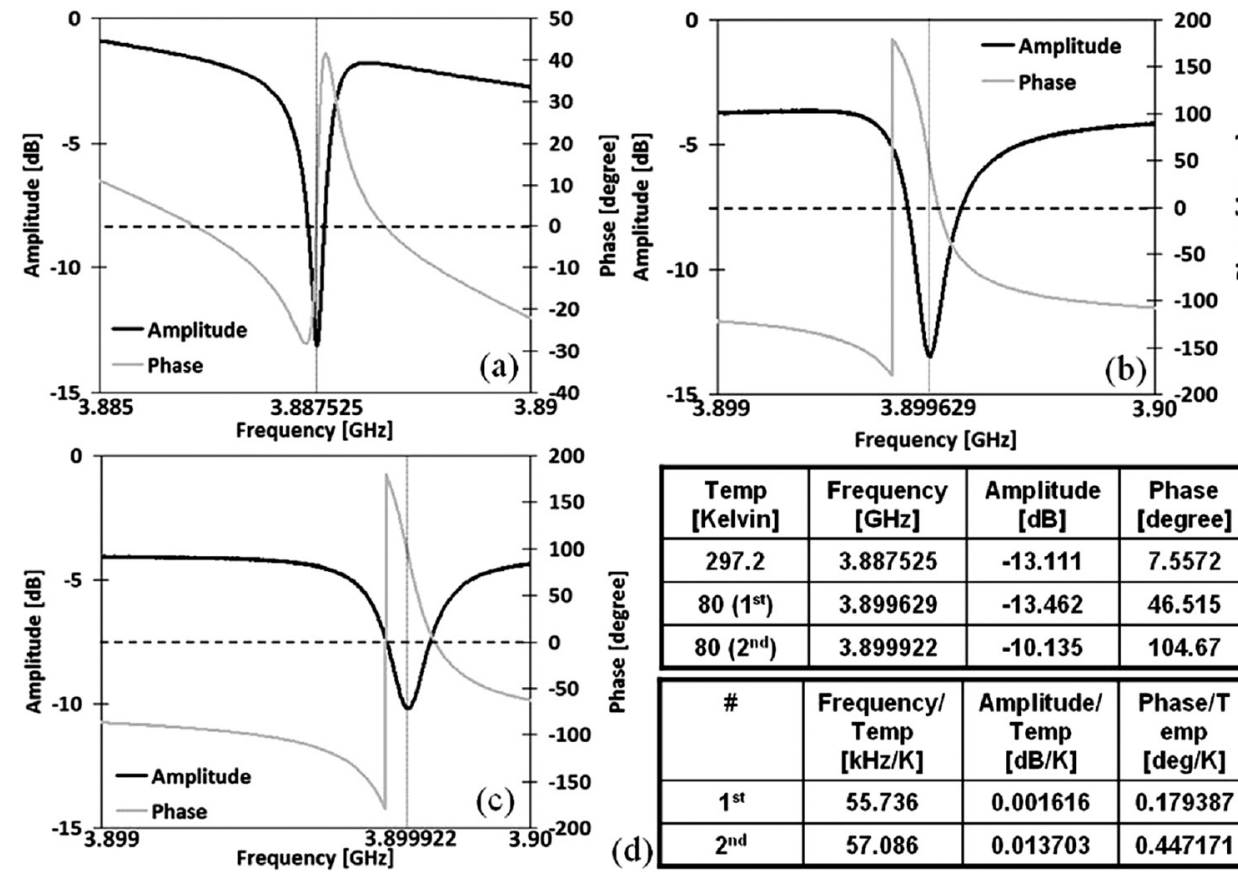

\begin{tabular}{|c|c|c|c|}
\hline $\begin{array}{c}\text { Temp } \\
\text { [Kelvin] }\end{array}$ & $\begin{array}{c}\text { Frequency } \\
\text { [GHz] }\end{array}$ & $\begin{array}{c}\text { Amplitude } \\
\text { [dB] }\end{array}$ & $\begin{array}{c}\text { Phase } \\
\text { [degree] }\end{array}$ \\
\hline 297.2 & 3.887525 & -13.111 & 7.5572 \\
\hline $80\left(1^{\text {st }}\right)$ & 3.899629 & -13.462 & 46.515 \\
\hline $80\left(2^{\text {nd }}\right)$ & 3.899922 & -10.135 & 104.67 \\
\hline$\#$ & $\begin{array}{c}\text { Frequency/ } \\
\text { Temp } \\
{[\mathrm{kHz} / \mathrm{K}]}\end{array}$ & $\begin{array}{c}\text { Amplitude/ } \\
\text { Temp } \\
\text { [dB/K] }\end{array}$ & $\begin{array}{c}\text { Phase/T } \\
\text { emp } \\
\text { [deg/K] }\end{array}$ \\
\hline $1^{\text {st }}$ & 55.736 & 0.001616 & 0.179387 \\
\hline $2^{\text {nd }}$ & 57.086 & 0.013703 & 0.447171 \\
\hline
\end{tabular}

FIG. 16. Experimental $\mathrm{S}_{11}$-spectal data of the 5 cell test cavity measured at (a) room temperature $(\mathrm{T}=297.2 \mathrm{~K})$, and $\mathrm{LN}_{2}$-cooled temperature $(\mathrm{T}=80 \mathrm{~K})$ (b) 0-day and (c) 20 days after cooling started. 
matching condition of the input coupler and non-uniform cell-to-cell field distribution. The cavity design in the deflecting performance can thus be improved by optimizing the coupling structure and cavity geometry. Volumetric change of the cavity dimensions resulting from $\mathrm{LN}_{2}$-cooling should be embedded in the design process to determine the correct operating frequency. The drafted 5-cell vacuum-cryomodule design for the ASTA beamline application was studied with the batched multiple simulation process, including RF, thermal, and mechanical solvers. The combined simulation results showed that thermal fluctuation arising from excessive cavity RF-loading can cause severe structural deformation and frequency deviation with long pulse length, high repetition rate operation. Also, the analytic correction will need to be considered in the high-Q cavity design. The fully integrated analysis module will enable rapid assessment on non-transient RF-thermal response and the resultant mechanical stresses of a high- $Q$ RF-cryogenic system under a pulsed operation. Although instantaneous frequency shifts from periodic pulse loading are analyzed only by time transient simulations, this bundled analysis offers a simple way of readily implementing stationary thermal effects in the cavity design. This will improve fast engineering and fabrication processes of a highly sensitive RF cavity by quickly evaluating and compensating resident frequency deviations of RFloaded cryogenic operation. Currently, more systematic optimization of the sensitivity modeling analysis on the deflecting mode vacuum-cryomodule is under consideration in comparison with experimental data.

\section{ACKNOWLEDGMENTS}

We would like to acknowledge Philippe R. G. Piot for the support of the experiment. We also thank James Santucci, Jerry M. Makara, and Christopher Prokop for the technical support of the system setup for the experiment.
${ }^{1}$ An RF Separated Kaon Bean from the Main Injector: Superconducting Aspects, edited by D. A. Edwards, Editor Report TM-2060, Fermilab, Batavia, Illinois, November 1998.

${ }^{2}$ C. Hovater, G. Arnold, J. Fugitt, L. Harwood, R. Kazimi, G. Lahti, J. Mammosser, R. Nelson, C. Piller, and L. Turlington, LINAC96, Geneva, Switzerland (1996), p. 77.

${ }^{3} \mathrm{D}$. Alesini, "RF deflector-based sub-ps beam diagnostics: application to FELs and advanced accelerators," in Physics and Applications of High Brightness Electron Beams, Erice, Sicily, 2005 (World Scientific, 2006); see also J. T. Moody, P. Musumeci, M. S. Gutierrez, J. B. Rosenzweig, and C. M. Scoby, Phys. Rev. ST Accel. Beams 12, 070704 (2009).

${ }^{4}$ R. Akre, L. Bentson, P. Emma, and P. Krejcik, "A Transverse RF deflecting structure for bunch length and phase space diagnostics," in Proc. 2001 Particle Accelerator Conf., Chicago (2001), p. 2353.

${ }^{5}$ P. Piot, D. R. Douglas, and G. A. Krafft, Phys. Rev. ST Accel. Beams 6, 030702 (2003).

${ }^{6}$ Y. Orlov, C. M. O’Neill, J. J. Welch, and R. Sieman, in Proceeding of 1991 Particle accelerator Conferences, San Francisco CA (1991), p. 2838.

${ }^{7}$ M. Cornacchia and P. Emma, Phys. Rev. ST Accel. Beams 5, 084001 (2002).

${ }^{8}$ P. Emma, Z. Huang, K.-J. Kim, and P. Piot, Phys. Rev. ST Accel. Beams 9, 100702 (2006).

${ }^{9}$ K. Bishofberger, D. Edwards, H. Edwards, S. Nagaitsev, J. Santucci, S. Wang, I. Bohnet, R. Brinkmann, J.-P. Carneiro, K. Desler, K. Floettmann, P. Piot, M. Ferrario, Y. Sun, J. Corlett, S. Lidia, S. Wang, N. Barov, and C. Bohn, Flat Electron Beam Production at FNAL-A Status Report TU404, LINAC 2002.

${ }^{10}$ Y. -E Sun, P. Piot, K.-J. Kim, N. Barov, S. Lidia, J. Santucci, R. Tikhoplav, and J. Wennerberg, Phys. Rev. ST Accel. Beams 7, 123501 (2004).

${ }^{11}$ P. Piot, Y.-E Sun, and K.-J. Kim, Phys. Rev. ST Accel. Beams 9, 031001 (2006).

${ }^{12}$ J. Ruan, A. S. Johnson, A. H. Lumpkin, R. Thurman-Keup, H. Edwards, R. P. Fliller, T. W. Koeth, and Y. -E Sun, Phys. Rev. Lett. 106, 244801 (2011).

${ }^{13}$ Y.-E. Sun, P. Piot, A. Johnson, A. H. Lumpkin, T. J. Maxwell, J. Ruan, and R. Thurman-Keup, Phys. Rev. Lett. 105, 234801 (2010).

${ }^{14} \mathrm{~T}$. W. Koeth, "An observation of a transverse to longitudinal emittance exchange at the Fermilab A0 photoinjector," Ph.D. dissertation (Rutgers University, 2009).

${ }^{15}$ T. W. Koeth, A Copper 3.9 GHz TM110 Cavity for Emittance Exchange PAC2007 Albuquerque (2007).

${ }^{16}$ CST Microwave Studio Suit, 2012. 